\title{
Helicobacter pylori Infection and Related Gastrointestinal Diseases in Southeast Asian Countries: An Expert Opinion Survey
}

\author{
Duc Trong Quach ${ }^{1,2 *}$, Ratha-Korn Vilaichone ${ }^{3,4,5}$, Khien Van Vu ${ }^{6}$, Yoshio \\ Yamaoka $^{7,8}$, Kentaro Sugano ${ }^{9}$, Varocha Mahachai ${ }^{4,10}$
}

\begin{abstract}
Background: Helicobacter pylori (H. pylori) infection is currently considered as an infectious disease irrespective of symptoms and stage of disease. This study aimed to survey the impact of $H$. pylori infection and the current management approaches in Southeast Asian countries. Materials and methods: This is a survey among 26 experts from 9 Southeast Asian countries (Cambodia, Indonesia, Laos, Malaysia, Myanmar, Philippines, Singapore, Thailand and Vietnam), who attended a meeting to develop the ASEAN consensus on H. pylori management in November 2015. Results: The prevalence of $H$. pylori varied significantly from $20 \%$ to $69 \%$ among countries, highest in Myanmar and lowest in Malaysia. The rate of $H$. pylori infection in patients with gastritis, peptic ulcer disease and gastric cancer (GC) also varied significantly, not only among countries but also among regions within the same country. The most common method for H. pylori diagnosis before treatment was rapid urease test, followed by urea breath test. In multi-ethnic countries, some ethnic groups including Chinese, Batak and Minahasanese were considered as having higher risk of GC. There have been no national screening programs for GC in all countries, and a majority of patients with GC were diagnosed in advanced stages with very poor 5-year survival. Conclusions: The prevalence of $H$. pylori infection and its infection rates in related gastrointestinal diseases were significantly different among Southeast Asian countries. The prognosis of patients with GC in the region was very poor. The result of this survey is a platform for future international and regional research collaboration.
\end{abstract}

Keywords: Helicobacter pylori- gastritis- peptic ulcer disease- gastric cancer- Southeast Asia

Asian Pac J Cancer Prev, 19 (12), 3565-3569

\section{Introduction}

It is well-known that Helicobacter pylori (H. pylori) infection causes chronic active gastritis in all colonized subjects (Malfertheiner et al., 2017). This bacterium is responsible for the majority of peptic ulcer disease as well as gastric cancer (GC) and has been classified as a type I carcinogen since 1994 (Uemura et al., 2001; Suerbaum and Michetti, 2002). At present, H. pylori infection is considered an infectious disease irrespective of an individual's symptoms and stage of disease as successful eradication cures gastritis and can alter the progression to long-term complications, or recurrence of disease(Sugano et al., 2015). Although H. pylori prevalence is decreasing in developed countries and some developing countries, the prevalence in Asia is estimated about $54.7 \%$, and there is a wide variation in prevalence between regions and countries (Hooi et al., 2017). The Southeast Asia region is critically important from the global health perspective as it suffers from high burdens of communicable and non-communicable diseases against a background of relatively poor health infrastructure (Narain and Bhatia, 2010). Despite of rapid economic growth and development during the last couple of decades, many countries in the region still have a high burden of communicable diseases (Gupta and Guin, 2010). However, there have been very limited data published in English about the current situation of $H$. pylori infection in countries belonging to

${ }^{1}$ Department of Internal Medicine, University of Medicine and Pharmacy at Hochiminh City, ${ }^{2}$ Department of Gastroenterology, Gia-Dinh's People Hospital, Hochiminh, ${ }^{6}$ Department of Gastrointestinal Endosccopy, 108 Central Military Hospital, Hanoi, Vietnam, ${ }^{3}$ Division of Gastroenterology, Department of Medicine, Thammasat University Hospital, ${ }^{4}$ National Gastric Cancer and Gastrointestinal diseases Research Center, ${ }^{5}$ Department of Medicine, Chulabhorn International College of Medicine (CICM) at Thammasat University, Pathumthani, ${ }^{10}$ GI and Liver Center, Bangkok Medical Center, Bangkok, Thailand, ${ }^{7}$ Department of Medicine, Gastroenterology Section, Baylor College of Medicine and Michael E. DeBakey VA Medical Center, Texas, USA, ${ }^{8}$ Department of Environmental and Preventive Medicine, Oita University Faculty of Medicine, Yufu, ${ }^{9}$ Department of Internal Medicine, Division of Gastroenterology, Jichi Medical University, Japan.*For Correspondence: drquachtd@ump.edu.vn 
the region and we are aware of some important data which have been reported in local languages. Therefore, expert survey is an important channel of information and may serve as a preliminary platform for further international and regional collaboration work.

\section{Materials and Methods}

This study was a survey among 26 experts from 9 Southeast Asian countries including Thailand, Cambodia, Indonesia, Laos, Malaysia, Myanmar, Philippines, Singapore and Vietnam who attended a meeting to develop the ASEAN consensus on $H$. pylori management in Bangkok in November 2015. The representative experts were invited based on their significant contributions to the field of $H$. pylori research in their countries and via collaboration activities among national societies of gastroenterology in the region. The experts' names and their affiliations are listed in the acknowledgements section. A questionnaire was sent to each member before the consensus meeting (Table 1). At the meeting, the collected information were presented, intensively discussed and then finalized by all experts.

\section{Results}

The proportion of area with limited health care facilities for $H$. pylori management in Southeast Asian countries significantly varied, ranging from less than $10 \%$ in Malaysia and Singapore, $10-50 \%$ in Laos, Indonesia and Thailand, $50-80 \%$ in Myanmar, Philippines and Vietnam and more than $80 \%$ in Cambodia. The prevalence of $H$. pylori infection was significantly different among countries and so were the rates of infection in related gastrointestinal diseases (Table 2). H. pylori prevalence was lowest in Malaysia (20\%) and highest in Myanmar $(68 \%)$.

Regarding the diagnostic methods before treatment, rapid urease test (RUT) was the most common method, followed by urea breath test (Table 3 ). The test-and-treat strategy for dyspeptic patients without alarming symptoms
Table 1. Questionnaire for Expert Opinion Survey on $H$. pylori Infection and Related Gastrointestinal Diseases in Southeast Asian Countries

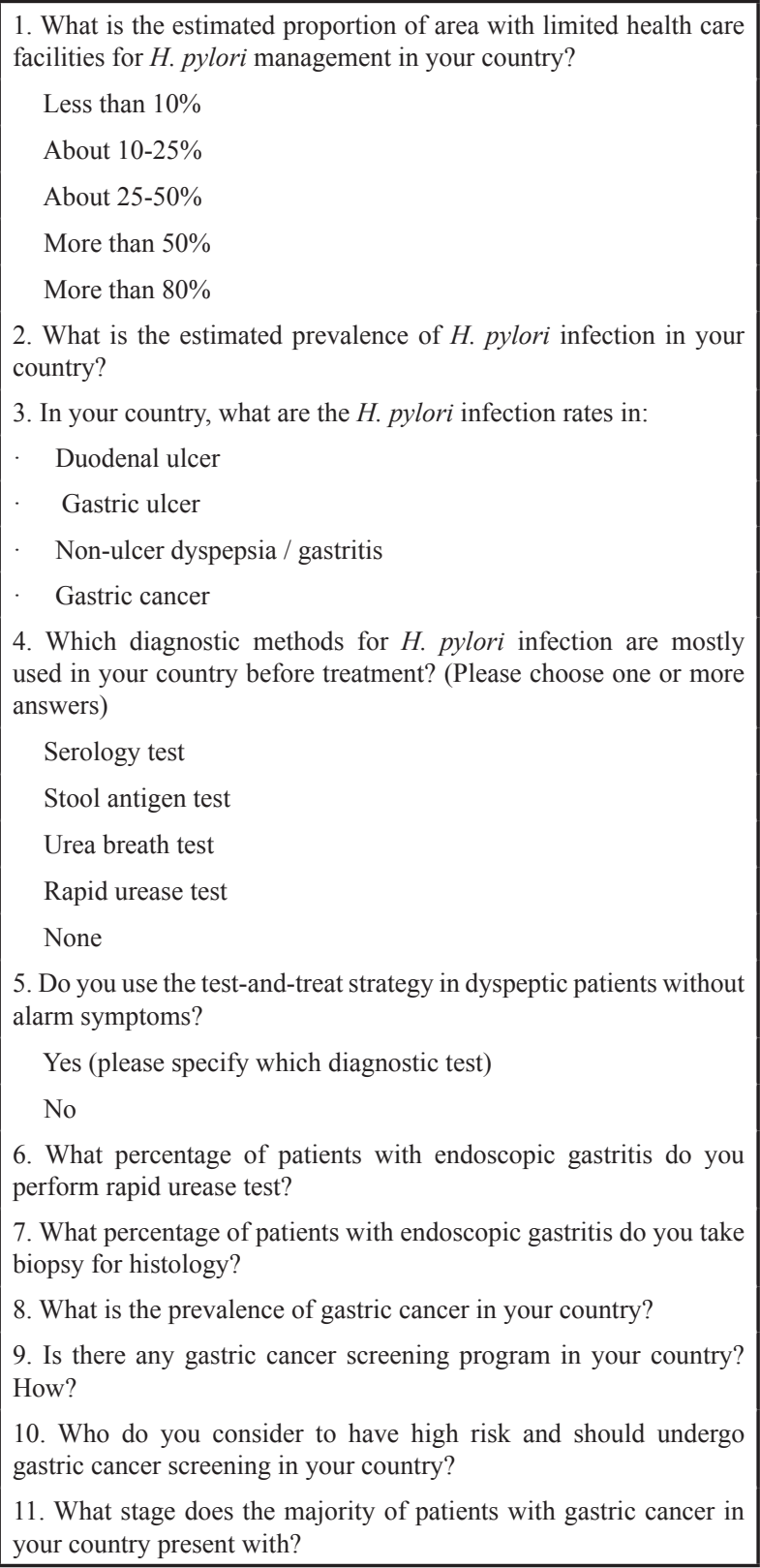

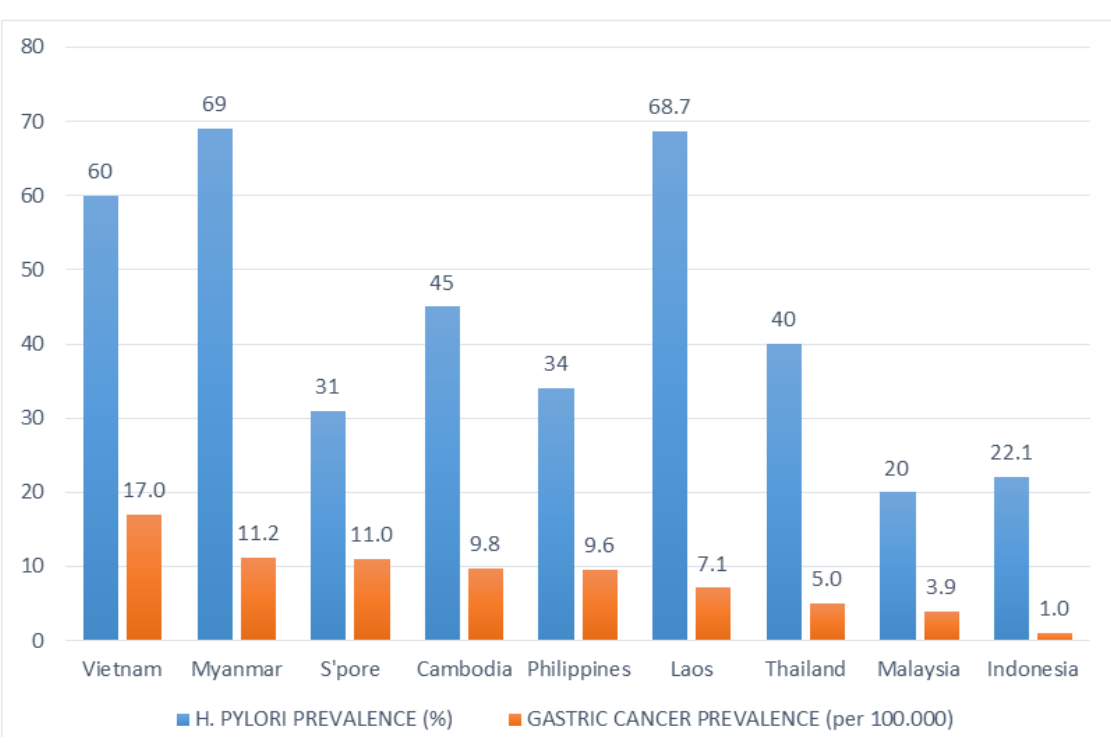

Figure 1. Estimated H. pylori and Gastric Cancer Prevalence in Southeast Asian Countries 
DOI:10.31557/APJCP.2018.19.12.3565

H. pylori Infection in Southeast Asia: an Expert Survey

Table 2. Estimated H. pylori Prevalence and the Infection Rates in Related Gastrointestinal Diseases in Southeast Asian Countries

\begin{tabular}{lccccccccc}
\hline Country & Cambodia & Indonesia & Laos & Malaysia & Myanmar & Philippines & Singapore & Thailand & Vietnam \\
\hline H. pylori prevalence (\%) & 45 & 22.1 & 68.7 & 20 & 69 & 34 & 31 & 40 & 60 \\
FH. pylori infection rates in related gastrointestinal diseases & & & & & & & \\
NUD/gastritis (\%) & 45 & 60 & 90 & 29 & 33.3 & 49.1 & NA & $23.0-61.0$ & 54 \\
Duodenal ulcer (\%) & 90 & 90 & 79 & 69.8 & 57.1 & 33.5 & NA & $48.0-90.0$ & 92.5 \\
Gastric ulcer (\%) & 70 & 80 & 78 & 56.8 & 57.1 & 36.5 & NA & $39.0-80.0$ & 84 \\
Gastric cancer (\%) & NA & NA & 16 & 37 & $>20.0$ & 36 & NA & $34.0-90.0$ & 80 \\
\hline
\end{tabular}

NUD, Non-ulcer dyspepsia, NA, data not available, $\uparrow$, Different responses among Thai experts

Table 3. Practical Approaches to H. pylori Diagnosis and Management in Southeast Asian Countries

\begin{tabular}{|c|c|c|c|c|c|c|c|c|c|}
\hline Country & Cambodia & Indonesia & Laos & Malaysia & Myanmar & Philippines & Singapore & Thailand & Vietnam \\
\hline \multicolumn{10}{|c|}{ Most common test for $H$. pylori diagnosis before treatment } \\
\hline Serology & + & + & - & + & - & + & - & - & + \\
\hline Stool Ag test & + & - & - & + & + & + & - & - & - \\
\hline UBT & - & + & - & + & + & + & + & + & + \\
\hline RUT & + & + & + & + & + & + & + & + & + \\
\hline \multicolumn{10}{|c|}{ Test-and-Treat strategy using non-invasive test for $H$. pylori in patients without alarming symptoms } \\
\hline Test-and-treat & Yes & Yes & No & Yes & Yes & No & No & Yes/No† & Yes \\
\hline Type of test & Stool Ag test & UBT & - & UBT & Stool Ag test & - & - & UBT & UBT \\
\hline \multicolumn{10}{|c|}{ Practical approach for $H$. pylori diagnosis for patients with endoscopic gastritis } \\
\hline RUT (\%) & 10 & $\ddagger$ & 30 & 100 & 90 & 100 & $>95.0$ & $>90.0$ & $>90.0$ \\
\hline Histology $(\%)$ & 100 & $\S$ & 70 & 100 & 50 & 10 & $>95.0$ & I & 10 \\
\hline
\end{tabular}

UBT, Urea breath test; RUT, Rapid urease test via upper gastrointestinal endoscopy; $\uparrow$ Majority of experts prefer not to use the test-and-treat strategy in Thailand; $\$, 20 \%$ (government hospital) and $60-80 \%$ (private hospital); $\S, 80 \%$ (government hospital) and $20-90 \%$ (private hospital); I, Significant varies responses from $0 \%$ to $100 \%$.

was applied in most Southeast Asian countries except for Laos and Philippines. Stool antigen and urea breath tests were commonly used for this purpose. For patients undergoing upper gastrointestinal endoscopy who had the findings of endoscopic gastritis, RUT and histology were performed with significantly different proportions among countries as well as among health centers and regions within the same country. In Indonesia, the practical approaches were different between government and private hospitals with more histology examinations performed in the private settings, which was partially explained by the reimbursement policy in this country.

The prevalence of GC in each country in comparison with the $H$. pylori prevalence in each population is summarized in Figure 1. It was highest in Vietnam (17/100.000) and lowest in Indonesia (1/100.000). Familial history of GC and precancerous gastric lesions were commonly considered as high-risk factors of GC development (Table 4). In multi-ethnic countries, some ethnic groups including Chinese, Batak and Minahasanese

Table 4. Current Screening Program, Surveillance Strategy and Prognosis of Gastric Cancer in Southeast Asian Countries

\begin{tabular}{|c|c|c|c|c|c|c|c|c|c|}
\hline Country & Cambodia & Indonesia & Laos & Malaysia & Myanmar & Philippines & Singapore & Thailand & Vietnam \\
\hline National screening program & No & No & No & No & No & No & No & No & No \\
\hline \multicolumn{10}{|c|}{ Gastric cancer stage and prognosis } \\
\hline $\begin{array}{l}\text { Stage at diagnosis in } \\
\text { majority of patients }\end{array}$ & Advanced & Advanced & Advanced & Advanced & Advanced & Advanced & Advanced & Advanced & Advanced \\
\hline 5 -year survival rate $(\%)$ & NA & $<10.0$ & NA & NA & NA & 11.6 & 26 & $<20.0$ & $` 10.0$ \\
\hline \multicolumn{10}{|c|}{ Individuals considered as high-risk patients of gastric cancer and should undergo screening } \\
\hline Ethnicity & - & $+\mathrm{a}$ & - & $+\mathrm{b}$ & - & - & - & - & - \\
\hline Elderly & - & - & + & - & + & - & - & + & + \\
\hline Precancerous GC lesions & - & - & + & - & - & + & + & + & + \\
\hline Family history of GC & + & - & + & - & + & + & - & + & + \\
\hline H. pylori infection & - & - & - & - & - & + & - & - & - \\
\hline Gastric ulcer & + & - & + & - & - & - & - & - & - \\
\hline Hx of gastrectomy & - & - & + & - & - & - & - & - & - \\
\hline Pepsinogen test $(+)$ & - & - & - & - & - & - & - & - & - \\
\hline
\end{tabular}


were considered as having higher risk. However, there have been no national screening programs for GC in all countries, and a majority of patients with GC were diagnosed in advanced stages with very poor 5-year survival.

\section{Discussion}

Our study showed that the prevalence of $H$. pylori infection was strikingly different among countries in the Southeast Asian region. Besides the genetic diversity of $H$. pylori, environmental factors (i.e. socioeconomic status, diet and smoking) and host genetic susceptibility have been reported to be related to the infection outcome (Correa and Piazuelo, 2008). Multi-ethnicity is one of the common and interesting characteristics of many countries in the region. Recent collaboration studies among Southeast Asian countries have shed light on the interactions among these above-mentioned factors. Population-based cross-sectional studies in Indonesia and Vietnam showed that variations in geographical region and ethnicity were independent factors associated with the risk of H. pylori acquisition (Syam et al., 2015; Binh et al., 2018).

This survey showed that the rates of $H$. pylori infection in relation to upper gastrointestinal diseases were also significantly different among countries in the region (Table 2). The infection rate in patients with peptic ulcer disease was about one third in Philippines, two third in Malaysia, and more than two third of patients in the rest countries with available data. A previous meta-analysis in Southeast Asian countries showed that patients infected with vacA $\mathrm{m} 1$ type or cagA-positive strains had an increased risk of peptic ulcer disease and there was a significant variation in the frequencies of cagA status and vacA genotypes among countries and the individual races residing within each respective country (Sahara et al., 2012). A recent study in Vietnam, a multi-ethnic country in the region, showed that the E-De ethnic group living in the high plateau region of central Vietnam carried strains with Western-type CagA $(82 \%)$ and exhibited significantly lower gastric mucosal inflammation compared to other ethnic groups. But the histological scores of Western-type CagA and East-Asiantype CagA within the E-De group showed no significant differences (Binh et al., 2018). Therefore, host factors are likely to be important determinants in addition to bacterial virulence factors for gastric mucosal inflammation and contribute to the Asian enigma.

Regarding GC, Vietnam has the highest prevalence of GC among Southeast Asian countries. The majority (97\%) of strains in Vietnam was East-Asian type cagA, whereas only about half of the strains in Thailand and Malaysia was East-Asian type cagA and $94.1 \%$ of the strains in Myanmar was Western-type cagA strains (Sahara et al., 2012; Myint et al., 2018). One another interesting result was the significantly high proportion of $H$. pylori-negative GC. The H. pylori infection rate in patients with $\mathrm{GC}$ was reported $16 \%$ in Laos, $80 \%$ in Vietnam and raging from 34 to $90 \%$ in Thailand. This could raise a concern about the reliability of diagnostic methods for $H$. pylori infection. The worldwide proportion rate of $H$. pylori-negative GC according to recent reports is about $0.4-5.4 \%$ (Yamamoto et al., 2015). The minimum criteria for this diagnosis include negative findings in two or more methods (endoscopic, pathological, serum pepsinogen test), negative UBT or serum IgG test and no history of $H$. pylori eradication (Yamamoto et al., 2015). There have been so far no studies in the Southeast Asian region applying the strict criteria. The data were collected from experts' experience during their clinical practice with great variants. Two recent studies used a combination of several tests (culture, histology, RUT and serum $H$. pylori antibody test) showed that $17.0 \%$ of GC patients in Thailand and $20.6 \%$ of GC patients in Vietnam had negative results for all four tests (Vilaichone et al., 2014; Binh et al., 2017). In addition, the rate of unintended $H$. pylori eradication in the Vietnamese was about $11 \%$, which is similar to that in the Japanese (Ono et al., 2012; Boda et al., 2014; Hiyama et al., 2015). Therefore, the rate of $H$. pylori-negative $\mathrm{GC}$ in the region may be higher compared to the literature. Other environmental factors may play important roles in the development of GC, which should be investigated by applying the strict criteria.

This study has some limitations. First, it is a survey of expert opinions and there are limited strong evidence to support the data. Second, the methodology for the diagnosis of $H$. pylori infection used in local studies could be heterogeneous and, therefore, may not reflect the true picture.

In summary, there were significant differences in $H$. pylori acquirement and the infection rates in patients with gastritis, peptic ulcer diseases and GC among Southeast Asian countries. No countries in the region have national screening program for $\mathrm{GC}$, and the prognosis of patients with GC is very poor. The result of this survey is a platform for future international and regional research collaboration.

\section{Acknowledgements}

This study was partially supported by National Gastric Cancer and Gastrointestinal diseases Research Center (NRGC) of Thailand, Gastroenterology Association of Thailand (GAT), Ministry of public health (Thailand) and the Asian Pacific Association of Gastroenterology (APAGE). We acknowledge the input information from the following 26 experts from ASEAN countries:

- Varocha Mahachai (Department of Medicine, Faculty of Medicine, Chulalongkorn University and King Chulalongkorn Memorial Hospital and National Gastric Cancer and Gastrointestinal Diseases Research Center, Bangkok, Pathumthani, Thailand)

- Somchai Leelakusolvong (Department of Medicine, Siriraj Hospital, Mahidol University, Thailand)

- Ratha-korn Vilaichone (Department of Medicine, Thammasat University Hospital, Khlong Luang and National Gastric Cancer and Gastrointestinal Diseases Research Center, Bangkok, Pathumthani, Thailand. Department of Medicine, Chulabhorn International College of Medicine (CICM) at Thammasat University, Pathumthani, Thailand.)

- Monthira Maneerattanaporn (Department of 
Medicine, Siriraj Hospital, Mahidol University and National Gastric Cancer and Gastrointestinal Diseases Research Center, Bangkok, Pathumthani, Thailand)

- Sombat Treeprasertsuk (Department of Medicine, Faculty of Medicine, Chulalongkorn University, Thailand)

- Chomsri Kositchaiwat (Department of Medicine, Ramathibodi Hospital, Mahidol University, Thailand)

- Alex Leow (Division of Gastroenterology and Hepatology, Department of Medicine, University of Malaya, Kuala Lumpur, Malaysia)

- Swe Mon Mya (Department of Gastroenterology, Yangon General Hospital, Yangon, Myanmar)

- Oung Chakravuth (Calmette Hospital, University of Health Science, Phnom Penh, Cambodia)

- Rapat Pittayanon (Department of Medicine, Faculty of Medicine, Chulalongkorn University and King Chulalongkorn Memorial Hospital and National Gastric Cancer and Gastrointestinal Diseases Research Center, Bangkok, Pathumthani, Thailand)

- Jarin Rojborwonwitaya (Department of Medicine, Thonburi Hospital, Thailand)

- Peranart Chotivitayatarakorn (Department of Medicine, Thammasat University Hospital, Khlong Luang and National Gastric Cancer and Gastrointestinal Diseases Research Center, Bangkok, Pathumthani, Thailand)

- Pises Pisespongsa (Department of Medicine, Bumrungrad Hospital, Thailand)

- Pisaln Mairiang (Department of Medicine, Faculty of Medicine, KhonKaen University, Khon Kaen, Thailand)

- Sengdao Vannarath (Department of Gastroenterology, Mahosot Hospital, Vientiane, Laos)

- Moe Myint Aung (Department of Gastroenterology, Yangon General Hospital, Yangon, Myanmar)

- Tiing-Leong Ang (Department of Gastroentrology and Hepatology, Changi General Hospital, Singapore)

- Bouachanh Rasachak (Department of Gastroenterology, Mahosot Hospital, Vientiane, Laos)

- Jose D Sollano (Section of Gastroenterology, University of Santo Tomas Hospital, Manila, Philippines)

- Duc Trong Quach (Department of Internal Medicine, University of Medicine and Pharmacy, Hochiminh City, Vietnam)

- Inchaya Sansak (Udonthani Hospital, Udon Thani, Thailand)

- Khien Van Vu (Department of Gastrointestinal Endosccopy, 108 Central Military Hospital, Hanoi, Vietnam)

- Piyathida Harnsomburana (Department of Medicine, Rajavithi Hospital, Bangkok, Thailand)

- Kwong-Ming Fock (Faculty of Medicine, National University of Singapore, Singapore)

- Khean-Lee Goh (Division of Gastroenterology and Hepatology, Department of Medicine, University of Malaya, Kuala Lumpur, Malaysia)

- Ari Fahrial Syam (Division of Gastroenterology, Department of Internal Medicine, Faculty of Medicine, University of Indonesia, Depok, Indonesia).
H. pylori Infection in Southeast Asia: an Expert Survey

\section{References}

Binh TT, Tuan VP, Dung HDQ, et al (2017). Advanced non-cardia gastric cancer and Helicobacter pylori infection in Vietnam. Gut Pathog, 9, 46.

Binh TT, Tuan VP, Dung HDQ, et al (2018). Molecular epidemiology of Helicobacter pylori infection in a minor ethnic group of Vietnam: A multiethnic, population-based study. Int J Mol Sci, 19, 708 .

Boda T, Ito M, Yoshihara M, et al (2014). Advanced method for evaluation of gastric cancer risk by serum markers: determination of true low-risk subjects for gastric neoplasm. Helicobacter, 19, 1-8.

Correa P, Piazuelo MB (2008). Natural history of Helicobacter pylori infection. Dig Liver Dis, 40, 490-6.

Gupta I, Guin P (2010). Communicable diseases in the South-East Asia Region of the World Health Organization: towards a more effective response. Bull World Health Organ, 88, 199-205.

Hiyama T, Quach DT, Le QD, et al (2015). Rate of unintended Helicobacter pylori eradication in the Vietnamese. Helicobacter, 20, 156-7.

Hooi JKY, Lai WY, Ng WK, et al (2017). Global prevalence of Helicobacter pylori infection: Systematic review and meta-analysis. Gastroenterology, 153, 420-9.

Malfertheiner P, Megraud F, O'Morain CA, et al (2017). Management of Helicobacter pylori infection-the Maastricht V/Florence Consensus Report. Gut, 66, 6-30.

Myint T, Miftahussurur M, Vilaichone RK, et al (2018). Characterizing Helicobacter pylori cagA in Myanmar. Gut Liver, 12, 51-7.

Narain JP, Bhatia R (2010). The challenge of communicable diseases in the WHO South-East Asia Region. Bull World Health Organ, 88, 162.

Ono S, Kato M, Suzuki M, et al (2012). Frequency of Helicobacter pylori -negative gastric cancer and gastric mucosal atrophy in a Japanese endoscopic submucosal dissection series including histological, endoscopic and serological atrophy. Digestion, 86, 59-65.

Sahara S, Sugimoto M, Vilaichone RK, et al (2012). Role of Helicobacter pylori cagA EPIYA motif and vacA genotypes for the development of gastrointestinal diseases in Southeast Asian countries: a meta-analysis. BMC Infect Dis, 12, 223.

Suerbaum S, Michetti P (2002). Helicobacter pylori infection. $N$ Engl J Med, 347, 1175-86.

Sugano K, Tack J, Kuipers EJ, et al (2015). Kyoto global consensus report on Helicobacter pylori gastritis. Gut, 64, 1353-67.

Syam AF, Miftahussurur M, Makmun D, et al (2015). Risk factors and prevalence of Helicobacter pylori in five largest islands of Indonesia: A preliminary study. PLoS One, 10, e0140186.

Uemura N, Okamoto S, Yamamoto S, et al (2001). Helicobacter pylori infection and the development of gastric cancer. N Engl J Med, 345, 784-9.

Vilaichone RK, Panarat W, Aekpongpaisit S, et al (2014). Clinical characteristics and Helicobacter pylori status of gastric cancer in Thailand. Asian Pac J Cancer Prev, 15, 9005-8.

Yamamoto Y, Fujisaki J, Omae M, et al (2015). Helicobacter pylori-negative gastric cancer: characteristics and endoscopic findings. Dig Endosc, 27, 551-61.

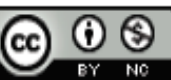

This work is licensed under a Creative Commons AttributionNon Commercial 4.0 International License.

Asian Pacific Journal of Cancer Prevention, Vol 19 